\title{
Recent advances and open challenges in percolation
}

\section{Review Article}

Author(s):

Araújo, N.; Grassberger, Peter; Kahng, Byungnam; Schrenk, K.J.; Ziff, Robert M.

Publication date:

2014

Permanent link:

https://doi.org/10.3929/ethz-b-000097888

Rights / license:

In Copyright - Non-Commercial Use Permitted

Originally published in:

The European Physical Journal Special Topics 223(11), https://doi.org/10.1140/epjst/e2014-02266-y 


\title{
Recent advances and open challenges in percolation
}

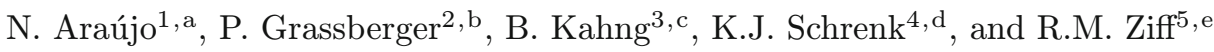 \\ 1 Departamento de Física, Faculdade de Ciências, Universidade de Lisboa, 1749-016 \\ Lisboa, Portugal, and Centro de Física Teórica e Computacional, Universidade de \\ Lisboa, 1749-016 Lisboa, Portugal \\ 2 JSC, FZ Jülich, 52425 Jülich, Germany \\ 3 Department of Physics and Astronomy, Seoul National University, Seoul 151-747, Korea \\ ${ }^{4}$ Computational Physics for Engineering Materials, IfB, ETH Zurich, \\ Wolfgang-Pauli-Strasse 27, 8093 Zurich, Switzerland and Department of Chemistry, \\ University of Cambridge, Lensfield Road, Cambridge CB2 1EW, UK \\ ${ }^{5}$ Center for the Study of Complex Systems and Department of Chemical Engineering, \\ University of Michigan, Ann Arbor, Michigan 48109-2136, USA
}

Received 22 April 2014 / Received in final form 18 August 2014

Published online 24 October 2014

\begin{abstract}
Percolation is the paradigm for random connectivity and has been one of the most applied statistical models. With simple geometrical rules a transition is obtained which is related to magnetic models. This transition is, in all dimensions, one of the most robust continuous transitions known. We present a very brief overview of more than 60 years of work in this area and discuss several open questions for a variety of models, including classical, explosive, invasion, bootstrap, and correlated percolation.
\end{abstract}

\section{Introduction}

Percolation is a classic problem in statistical physics and, like a cat with nine lives, never seems to die. The study by its proper name began with the work of engineer Simon Broadbent and mathematician John Hammersley in the 1950's [1], inspired by the workings of activated charcoal gas masks, but it was effectively already considered by chemist Paul Flory in the early 1940's in his study of gelation in polymers [2-4]. The King's College London group of Cyril Domb, which included Michael Fisher, John Essam, and M.F. Skyes, did a great deal to popularize the percolation problem in the physics community starting in the 1960's [5]. In an early paper, Fisher and Essam showed that the polymerization model of Flory corresponds to percolation

\footnotetext{
a e-mail: nmaraujo@fc.ul.pt

b e-mail: p.grassberger@fz-juelich.de

c e-mail: bkahng@snu.ac.kr

d e-mail: kjs73@cam.ac.uk

e e-mail: rziff@umich.edu
} 
on the Bethe lattice [6,7]. There followed a great deal of work by physicists, including Rushbrooke, Stanley, Coniglio, Halperin, Herrmann, Stauffer, Aharony, Havlin, Duplantier, etc. [8-10]. A major breakthrough was the demonstration in 1969 by Fortuin and Kasteleyn [11] that percolation is a limiting case of the general Potts model, which includes the Ising model, and can be solved exactly. This paved the way to many exact results in percolation (e.g., for scaling relations [12-14], the hull [15-19], and the number of red bonds [20]), and allowed powerful renormalization group ideas to be used [21]. Numerous variations of percolation, such as invasion percolation, first-passage percolation, directed percolation, and bootstrap percolation were introduced. Exact "static" exponents in 2d were first proposed in the 1980's by den Nijs [22-24], Pearson and others, but attempts to find exact exponents for conductivity (the Alexander-Orbach conjecture) [25], the backbone [26,27], and the shortest path (chemical distance) [28] have not succeeded.

Finding percolation thresholds both exactly and by simulation has been an enduring subject of research in this field (see [29] and references therein), as well as the development of algorithms such as those by Hoshen and Kopelman [30], by Leath [31], and by Newman and Ziff [32]. Finding rigorous proofs of exact thresholds and bounds has also been an enduring area of research for mathematicians (Kesten [33], Wierman [34], Grimmett [35], Bollobás and Riordan [36], etc.).

The derivation of an exact formula for the crossing probability on a rectangle by Cardy in 1992 [37] (motivated by numerical work of Langlands et al. [38]) led to a great deal of work on universal crossing properties in two-dimensional system, such as those by Pinson [39], Watts [40], Simmons [41], etc. Around 2000, Schramm developed the Stochastic Loewner Evolution (SLE) theory, which was soon applied to percolation hulls $[19,42,43]$. This caused once again percolation to be an active area of research in mathematical probability theory, and led to two Fields' medals, to Stanislav Smirnov and Wendelin Werner $[18,44,45]$. The results include rigorous derivation of the static percolation exponents. Another infusion of interest in percolation came from the surging field of network theory, which goes back to the study of random and complete graphs by Erdös and Rényi (1959), where the formation of a "giant component" is exactly analogous to percolation [46], but was revitalized by interest in small-world and scale-free networks. In 2000, Newman and Moore found the critical point for a random graph in the limit of large size, in which case the system is effectively a Bethe lattice, and this result connects to the early work of Flory, Fisher and Essam, but here with a general degree distribution [47]. In the field of random networks, the model of explosive percolation (EP) was first introduced by Achlioptas, D'Souza and Spencer [48], and this has been another problem that has led to a wave of new interest in percolation.

In this article, we present some perspective on open problems and challenges in percolation. The field is so vast that we can only touch on a subset of them, more aligned with our own interests.

\section{Classical uncorrelated percolation}

The most basic question in percolation is the value of the threshold for a given system. Exact thresholds in two dimensions for the square, triangular, honeycomb and related lattices (kagomé and $\left(3-12^{2}\right)$ site thresholds) were found by Sykes and Essam in 1963, using the star-triangle transformation [49]. In 1984 Wierman [34] generalized this transformation to find the threshold of the bow-tie lattice, and in 2006 Ziff and Scullard [50] showed that thresholds can be found for any lattice that can be represented as a self-dual 3-hypergraph (that is, decomposed into triangles that form a self-dual arrangement). Just recently, Grimmett and Manolescu [51] showed 
that thresholds can be found for any lattice that can be represented geometrically as an isoradial graph, yielding a broad new class of exact thresholds and providing a proof [52] of Wu's 1979 conjecture [53] for the threshold of the checkerboard lattice. So the widely held belief that there are just a handful of lattices where the threshold is known exactly is no longer true. However, many systems of long interest (such as site percolation on the square and honeycomb lattices, and bond percolation on the kagomé lattice) do not fit into the self-dual hypergraph or isoradial forms and cannot presently be solved. Is there another approach that can give exact thresholds to these and other lattices? Or can a proof be made that thresholds for some lattices can never be found in a closed form? New methods to obtain very high precision estimates of the threshold with unprecedented precision (over 12 digits for some models) have been developed by Jacobsen and Scullard [54,55], but none of these seem to suggest any obvious closed-form values. For higher dimensions, exact thresholds would be very beneficial but seem unlikely. In four dimensions, a claim for an exact threshold of plaquettes has been made [56].

Likewise, there are no exactly known exponents for more dynamical properties such as the shortest-path exponent $d_{\text {min }}$, dynamic exponent $z$, conductivity exponent $t$, spectral dimension, etc., even in two dimensions (only the related exponent $g$ is known $[57,58])$. For directed percolation, which can be interpreted as a dynamical form of percolation also related to the contact process, no exact results are known for any dimension. After years of trying, it seems unlikely that these other exponents will ever be found exactly, but perhaps a new breakthrough will be found.

Many questions relating to scaling functions and amplitude ratios were looked at extensively in the 1980's, but several questions remain unanswered, and with new computers and algorithms these problems are worth revisiting [59]. Amplitude ratios are useful for confirming universality of different percolation models [60,61]. Near the percolation threshold the size distribution of percolation clusters satisfies the scaling form $n_{\mathrm{s}}(p) \sim A s^{-\tau} f\left(B\left(p-p_{\mathrm{c}}\right) s^{\sigma}\right)$ where $A$ and $B$ are system-dependent scale factors, while $\tau$ and $\sigma$ are universal exponents and $f(z)$ is an universal function for a given dimensionality. The universality of $f(z)$ implies the universality of amplitude ratios (such as the ratio of the mean cluster size an $\epsilon$ above and below $p_{c}$ ), which were studied extensively in the past [62]. With a precise determination of the scaling function, many of these universal ratios should be able to be determined, but as far as we know the scaling function has never been determined precisely and used for this. For the correlation-length $\xi$, the amplitude ratio has been predicted to be exactly 2 [63], but because of questions of how the correlation length should be measured, it seems that this value has never been verified numerically. In 2001, Seaton [64] predicted an exact amplitude ratio for percolation, $\tilde{R}_{\xi}^{+}=\left[\alpha(2-\alpha)(1-\alpha) \tilde{A}^{+}\right]^{1 / d} \xi_{0}^{+}=$ $[40 / 27 \sqrt{3}]^{\frac{1}{2}}=0.9248 \ldots$ where $\alpha=-2 / 3, \tilde{A}^{+}$is the coefficient of the singular part $\left|p-p_{\mathrm{c}}\right|^{2-\alpha}$ of the free energy (number of clusters) and $\xi_{0}^{+}$is the coefficient to the divergence of the correlation length $\xi \sim \xi_{0}\left(p_{c}-p\right)^{-\nu}$ for $p<p_{c}$. While this ratio agreed with previous results by Delfino and Cardy [65], questions about the correct definition of the correlation function to use remain.

A way to put the size distribution into a universal form is by considering the enclosing area or volume; in that case the area of the $n$-th ranked cluster satisfies Zipf's law $A_{n} \sim C / n$, with $C$ known exactly in the case of two-dimensional enclosed area $[66,67]$. How this quantity behaves away from $p_{c}$ and how that relates to regular scaling functions has not been detailed. This same approach should apply to any critical system, including directed percolation.

An early exact result in percolation is the density $n$ of clusters of wet sites in bond percolation at the threshold, found by Temperley and Lieb [68] for the square lattice and evaluated simply as $(3 \sqrt{3}-5) / 2$ [69], and is also known for bond percolation on the triangular lattice [70]. Surprisingly, while many things have been proven exactly 
for site percolation on the triangular lattice, this quantity is known only numerically there: $n=0.0176255(5)$ [69]. Can this quantity also be found exactly?

\section{Classical percolation with long-range correlated disorder}

Percolation theory and related models have been applied to study transport and geometrical properties of disordered systems [71,72]. Among the first papers on correlated percolation is the work of Coniglio and Klein [73]. They have studied bond percolation on Ising clusters and found that in two dimensions the Ising critical point coincides with the percolation critical point for clusters of parallel spins.

Frequently, disorder is characterized by a power-law long-ranged spatial correlation. This fact has motivated studies of percolation models where the sites of the lattice are not occupied independently, but instead with long-range spatial correlation, in a process named correlated percolation [71,72,74-87]. The qualitative picture that emerged is that, in the presence of long-range correlations, percolation clusters become more compact and their transport properties change accordingly. These findings have also been confirmed by experimental studies of the transport properties of clusters in correlated invasion percolation $[88,89]$.

The typical strategy to investigate correlated percolation is to work with a landscape of random heights $h$, where $h(\mathbf{x})$ is the height of the landscape at the lattice site at position $\mathbf{x}[71,74-76,90,91]$. Recently, ranked surfaces have been introduced, providing the adequate framework to tackle this problem $[86,92]$. The ranked surface of a discrete landscape is constructed as follows: One first ranks all sites in the landscapes according to their height, from the smallest to the largest value. Then, a ranked surface is constructed where each site has a number corresponding to its position in the rank. The following percolation model can then be defined: Initially, all sites of the ranked surface are unoccupied. The sites are occupied one-by-one, following the ranking. At each step, the fraction of occupied sites $p$ increases by the inverse of the total number of sites in the surface. By this procedure, a configuration of occupied sites is obtained, the properties of which depend on the landscape. For example, if the heights are distributed uniformly at random, classical percolation with fraction of occupied sites $p$ is obtained $[32,93,94]$. To generate ranked surfaces with power-law correlated disorder the Fourier filtering method is used [75, 95-102].

The focus is usually on the dependence of percolation properties on how the correlations decay with the spatial distance, typically characterized by the Hurst exponent $H$. Schmittbuhl et al. have shown that for $H>0$, there is no percolation transition [76]. Instead, compact clusters grow and merge in such a way that the size of the largest cluster grows linearly with the fraction of occupied sites. By contrast, for $H<0$ the percolation transition is critical and critical exponents can be found. According to the extended Harris criterion, the critical exponents should change with $H[74,76,85,86,103,104]$. It was found that the fractal dimension of the largest cluster, its perimeter, as well as the dimension of its shortest path and backbone depend on $H[75,76,86,90,105]$. Strong dependence on $H$ is also found for the electrical conductivity exponent of the largest cluster and the growth of bridge sites in the correlated percolation model. Schrenk et al. have proposed a functional dependence of several exponents on the Hurst exponent $H$ [86]. While these relations were found as the simplest rational expressions giving the considered critical exponents as function of $H$, they do not have theoretical support. It is still an open question if these expressions can be found by scaling arguments or field theory. Concerning the perimeter fractal dimension of the largest cluster, it was found numerically that the duality relation of Duplantier [106] is fulfilled in the full range of Hurst exponents [86]. A more theoretical argument supporting this finding is also still missing. 


\section{Variants of percolation}

"Classical" (ordinary) percolation deals $[9,10]$ with the situation where small bridges are either established or removed randomly, irreversibly, and independently, until large scale connectivity is either established or lost. Here, a "bridge" might be a link or a node in a network, with regular lattices being very regular class of special networks. Apart from this no other processes of relevance go on, which means that each bridge is dealt with only once, whence the randomness or the phenomenon can be either considered as frozen (a view mostly taken in condensed matter) or as characterizing a stochastic contagion process, in which case it is describing the spreading of some epidemic [107], computer malware, or public opinion. In the latter context, it is also called the SIR ("susceptible-infected-removed") epidemic model $[61,108,109]$, to be contrasted to the SIS ("susceptible-infected-susceptible") model where bridges are removed after a finite life time (of infectivity), and which corresponds to directed percolation in space-time (the directness being that of the time arrow). Both SIR and SIS (i.e., undirected and directed percolation) show transitions between phases with and without long range connectivity, which are continuous ("second order") in the sense that a suitable order parameter (which usually is the density of the largest connected cluster) is zero at the transition point and increases continuously (but of course with singular derivatives) as one enters the supercritical (connected) phase. Deviations from this classical model can involve any of the basic ingredients:

1. "Bridges" (links, infections) themselves can already be long ranged. Models with power behaved long range interactions have been studied in statistical physics since the late 1960's, when it was realized that the 1-dimensional Ising model can have a non-trivial phase transition if interactions are sufficiently long ranged, with a decay $\sim 1 / r^{2}$ being the turning point [110-112]. Very early, Thouless [112] argued that the transition in this model is of Kosterlitz-Thouless [113] type, i.e. it is of infinite order, the correlation length does not scale with a power of the distance from the critical point, and there are generic power laws with continuously varying exponents in the subcritical phase. The same should be true for percolation. But his arguments were soon shown to be incomplete [111]. Indeed, when it was proven that in this case the transition is first order (discontinuous) [114], the predictions of Kosterlitz seemed to be obsolete. But recent simulations [115] show that there are indeed continuously varying critical exponents (in the supercritical phase), and that the correlation length diverges exponentially. Related to this is a model by Boettcher et al. [116], where long range links are not established in a completely random fashion. Rather, in the Boettcher et al. model all long range bonds must have length $2^{k}, k=1,2,3, \ldots$, bonds with length $2^{k}$ connect only to sites with coordinates $i \equiv 0 \bmod 2^{k}$, and they are all established with the same probability $p$. This leads to the same average density of long range links as in the random model with $P(r) \sim 1 / r^{2}$, and both models show very similar behavior. How similar they are in details remains to be seen.

2. In two dimensions, there is no similarity with the Kosterlitz-Thouless transition, but there is a long-standing controversy. When increasing the power in the contact probability $\sim 1 / r^{\sigma}$, there comes a point where the critical behavior changes from a regime with $\sigma$-dependent critical exponents to ordinary (short-range) percolation. The controversy deals with the question when this happens. Different field theoretical arguments suggest that it either happens when also the scaling of the supercritical process changes, or already for smaller $\sigma$. Extensive simulations (both for percolation and for the Ising model, see $[117,118]$ ) seemed to have settled this in favor of the latter option, but very recent simulations suggest that neither is right and things might be more complicated [119-121]. 
3. Bridges can be established in a not entirely random way. In particular, when establishing a new bridge, one may take two random (and not yet established) "candidates," and establish that candidate that leads to less long range connectivity (i.e., to a smaller cluster). This was proposed by Achlioptas et al. [48], who termed it "explosive percolation" and claimed it to have a discontinuous transition, where the transition is delayed so long that it finally takes place in a sudden, "explosive" way. This paper has led to several similar models, some of which actually do have first-order transitions (see e.g. $[122,123]$ ) - although the original Achlioptas model does not [124-126]. Notice that the choice between the two candidates introduces a severe non-locality which goes far beyond anything that can be described by a local field theory. It is presumably for this reason that the Achlioptas model seems to show (there is not yet any analytic proof) finite size scaling that is qualitatively different from that expected from the renormalization group [126]. This family of models is discussed in detail in Sect. 5.

4. Bridges can be established in a partly synchronized way. In ordinary bond percolation, links in a graph are randomly selected one after the other. In "agglomerative percolation" [127-129] this is replaced by the rule that a cluster is selected randomly, and then all links connected to it are established immediately. On non-bipartite lattices in two and three dimensions this is in the universality class of ordinary percolation, but not in one-dimensional lattices [128], on a class of trees [127], and on Erdős-Rényi graphs [130]. Notice that this model also introduces some non-locality (albeit much weaker than in the Achlioptas model), if very large clusters are chosen during late stages of the process. A special feature happens on bipartite lattices like the square and simple cubic lattice [129,131]. There, at the critical point also a $Z_{2}$ symmetry is spontaneously broken, which leads at least in two dimensions to a new universality class.

5. In the standard scenario, bridges are newly established in a pre-existing "virtual" network e.g., in bond percolation all nodes and all possible links are already defined, while in site percolation the new sites are chosen from a pre-existing set and all links are already established. In contrast to this, Callaway et al. [132] considered the case where links are established in a growing network. More precisely, each time a node is added to the existing network, also a link is added with probability $\delta<1$. In contrast to the Barabási-Albert model [133], where the new links are added so that nodes with higher degree get attached preferentially, new links are added completely at random. Again this leads to a Kosterlitz-Thouless type transition when $\delta$ passes through a critical value, but now the transition is (as the original KT transition) of infinite order instead of being discontinuous.

6. The establishment of bridges can be a cooperative effect. This seems to be the most interesting case. Models of this type have been studied as bootstrap percolation (or as the closely related $k$-core percolation) since the 1970's $[134,135]$, while co-epidemics (see below) are a major health hazard.

- In bootstrap percolation with index $m$ one considers a connected cluster on some graph, and removes recursively all nodes which have less than $m$ neighbors in the cluster. For $m=1$ nothing is done, as a connected graph has always at least one neighbor. For $m=2$ all leaves are removed in a first step. This might turn some nodes, which originally had $\geq 2$ neighbors, into leaves. They are removed in a second step. If this again turns nodes into leaves, they are removed again, etc. In this way one ends up either with an empty cluster, or with a cluster where all nodes have $\geq 2$ neighbors. In $k$-core percolation one starts with the cluster which contains all nodes and keeps at the end only the largest connected component, i.e. one ends up finally with the largest connected subgraph in which all nodes have degree at least $k$. Bootstrap percolation can show either continuous or discontinuous transitions, depending on the type of 
networks (lattice or random), and on the dimensionality when the network is a regular lattice. For details see [135].

- Instead of deleting "poor" nodes, let us now consider the opposite process of complex contagion $[136,137]$. Here nodes are attached to a growing cluster and nodes with more neighbors in the cluster are more likely to be attached. A possible application is political opinion spreading. We assume that no person is so eloquent that (s)he alone is able to convince his or her neighbor. But if there is already a small group of early adapters, then the combined argumentation of several of them is able to convince others with probability $q$. When people get convinced if and only if they have $\geq m$ convinced neighbors, this leads exactly to bootstrap percolation. More interesting, however, is the case where the chance $q_{m}$ to be finally convinced (or infected, or attached to the cluster, depending on the application) depends non-trivially on $m$, the number of neighbors in the attacking cluster. Take e.g. site percolation on a square lattice, where any site is incorporated with probability $p$ into a growing cluster, if it has at least one infected neighbor. In this case $q_{m}=p$ for all $m \geq 1$. In bond percolation, on the other hand, each site has the same chance $p$ to convince (or infect) any not yet convinced (or healthy) neighbor. A site with $m$ infected neighbors succumbs then with probability $q_{m}=p+(1-p) p+\ldots(1-p)^{m-1} p=1-(1-p)^{m}$. In general we expect $q_{m}$ (the probability that $m$ neighbors together will lead to infection) to grow with $m$. If this growth is moderate (as e.g. in bond percolation) we expect the transition to be continuous and in the universality class of ordinary percolation. This is no longer true if $q_{m}$ grows too fast. In that case one finds first-order transitions, where the epidemic (political opinion, fashion, computer virus, ...) either does not take off at all or takes off explosively. In between the first and second order regimes there is a tricritical point whose properties on finitedimensional lattices where studied by Jansen et al. by field theoretic methods [138].

- For random sparse (e.g. Erdős-Rényi) networks the tricritical point is particularly easy to find $[136,139]$ : If $q_{1}>0$, the transition from second to first order happens exactly when $q_{2}=2 q_{1}$, independent of its degree distribution.

- One important physical application of this model is to critically pinned driven interfaces in isotropic random media [140]. It is well known that such interfaces are often self-affine (i.e., show nontrivial scaling), while the bulk behind them is not fractal (as it would be for critical percolation). Thus in this problem the phase transition is actually hybrid, i.e. it combines a jump in the order parameter (the density of the infected phase) with non-trivial scaling related to the interface. The fact that any first order transition in $k$-core percolation is actually hybrid was stressed in particular in [141].

- Notice that interfaces in the present model have in general overhangs (as in real isotropic media, but in contrast to the standard model $[140,142]$ used to describe them). This has important consequences. First of all, such interfaces in $1+1$ dimension are always in the ordinary percolation universality class [139], as conjectured already in [143] (see, however, $[144,145]$ ). This is related to the proof of [146] that there cannot be first-order transitions in 2-dimensional random media. Secondly, it means that there will always be some holes left in a growing cluster, and these holes in general will show non-trivial scaling at the pinning point (as required by its hybrid nature). Finally, it might imply that the point where the zero temperature random field Ising model changes from being hysteresis-free to having hysteresis is not, as claimed in [147], a critical point with upper critical dimension $d_{c}=6$, but a tricritical point with $d_{c}=5$. The field theoretical predictions of [138] for this tricritical point were 
not well confirmed by the simulations of [139] for $d=3$, but they were very well confirmed for $d=4$, where they are supposed to be much more precise.

- Instead of cooperativity between attacking neighbors, we can also have cooperativity between different modes of attack. This refers in particular to cooperative co-epidemics or syndemics [148]. Take e.g. the examples of the 1918 Spanish flu and TB $[149,150]$, or of HIV and a host of other diseases like diabetes, hepatitis, TB and others [151,152]. In these cases one disease weakens the victim so much that the victim falls prey to the other one. A simple SIR type model for this with permanently increased susceptibility for the other disease was developed in [153]. Simulations of this model on random graphs and on lattices [154] show a host of different behaviors, depending on the topology of the graph but also on the detailed of the implementation (and, in one case, even on the initial conditions). But whenever a first-order transition is found, it is in general hybrid.

7. The last model we shall discuss here are interdependent networks. Consider e.g. a country like Italy with towns connected by a network of electric power lines and another network of computer connections. The latter are needed for controlling the power stations, while the former are needed to provide power to the computers. If some node fails, then this can lead to a huge cascade of failures as happened indeed in Italy on September 28, 2003. As shown by Buldyrev et al. in a seminal paper [155], this failure would have been a first-order transition, if it had happened on a locally loopless network like e.g. a sparse Erdős-Rényi network. But, as shown in [128], it still would be a continuous transition on a 2-dimensional regular lattice. Since Italy is 2-dimensional but not quite regular, it is unclear what should apply in this case. But in any case, in a series of papers (see e.g. [156-158]) several other types of interdependencies were studied. In all cases with first-order transitions, these are indeed again hybrid.

Finally, let us point out a possible connection between interdependent networks and cooperative co-epidemics. People have livers and lungs, and both can become sick. Someone with a liver disease will be more likely to get also a lung infection and vice versa. Let us now assume that liver and lung infections are both lethal. In this extreme case we are dealing exactly with two interdependent networks as described in [155]. Details of this correspondence have not yet been worked out.

\section{Explosive percolation}

Conventionally percolation transitions are known to be continuous. Recently, however, interest in discontinuous percolation transitions (DPT) has been triggered and boosted by i) the explosive percolation model [48] and ii) the cascading failure model in interdependent networks [155]. Actually the subject of DPT was initiated by the $k$-core percolation model $[134,135,159,160]$ a long time ago. However, the mechanism of the DPT arising in the $k$-core model was unusual, so that further studies of the DPT had not proceeded very much. The two models i) and ii) were designed for DPTs; however, the governing processes to the percolation transition and their mechanisms are different: cluster coagulation and fragmentation processes for the model i) and ii), respectively. Moreover, the order parameters are also different. Thus, we need to discuss the two problems separately, and find common features in a unified framework.

We first discuss the DPT driven by cluster merging processes. The EP model was introduced motivated by a mathematical invention, the so-called Achlioptas process: at each step, we take the one (among multiple options) which is the optimal one for 
the formation of a given target pattern. To generate a DPT in random graph, at each step, two pairs of nodes that are not connected yet are selected at random, and one pair of nodes among them is taken and connected by a new link, which is the one that produces a smaller size of connected cluster than the size of the cluster produced by the other option. Then, the growth of large clusters is suppressed and clusters with medium sizes are abundant. As the number of added links is increased, such medium-sized clusters merge and create a macroscopic-sized cluster, which emerges drastically at a delayed percolation threshold.

Following this pioneering paper [48], many related models have been introduced $[122,161-168]$. One of noticeable issues addressed in following works was that the EP transition in random graph is not indeed discontinuous but continuous in the thermodynamic limit. Actually, due to extremely slow convergence to asymptotic behavior as the system size is increased, whether the EP is indeed continuous or discontinuous was hard to be determined numerically and became a controversial issue. This claim was addressed firstly based on numerical results for a specific model [124]; however, since the claim was not firmly supported by analytic solution, the claim had not been firmly accepted first time. Later, based on the numerically observed fact that the average size of medium-sized cluster is not sufficiently large [163], a mathematical proof [125] was presented, which is the following: the number of clusters that participate in the merging process to generate a macroscopic-sized cluster is not sub-extensive to system size in order of magnitude, and thus, it cannot bring out a DPT, but a continuous percolation transition.

The EP transition in Euclidean space has been also extensively studied $[161,169]$. However, whether the percolation transition is discontinuous or continuous had not been clarified, either. In spite of such extensive studies, understanding of the EP transition in Euclidean space and on random graph in a unified scheme had been absent. For the continuous percolation transition case, the Erdős and Rényi model on random graph was regarded as the mean-field model of the ordinary percolation model. To resolve this goal for the DPT case, a model called the spanning cluster-avoiding (SCA) model was introduced [123]. In this model, the target pattern in the Achlioptas process is taken as a spanning cluster, which is actually standard in Euclidean space. This model used previous results, the scaling behavior of the number of bridge bonds above a percolation threshold. Here the bridge bonds are those that would form a spanning cluster if occupied [92]. Using the scaling formula for the bridge bonds, the percolation thresholds of the SCA model were calculated analytically for various numbers of potential links in the Achlioptas process. This analytic result leads to the following conclusion: the EP transition can be either continuous or discontinuous, depending on the number of multiple options, if the spatial dimension is less than the upper critical dimension, and is always continuous otherwise. Subsequently, it was concluded that the transition of the ordinary EP model is continuous as a mean-field solution of the SCA model.

Further intriguing and fundamental questions remain open. For example, the DPT occurring in the SCA model is rather trivial in the sense that the order parameter increases drastically all the way to unity at a trivial percolation threshold, which is equal to the system size in the thermodynamic limit. This behavior is similar to the one of the DPT governed by the Smoluchowski coagulation equation with the reaction kernel $k_{i} k_{j} \sim(i j)^{\omega}$ with $0 \leq \omega<0.5$ [170]. Therefore, it would be intriguing to study a non-trivial DPT model in which the order parameter is increased all the way to a finite value less than unity at a finite threshold, and gradually beyond that point [116,171-177]. Investigation of the origin of such a non-trivial DPT would be also interesting. Moreover, understanding of other discontinuous phase transitions in non-equilibrium systems such as synchronization transitions [178] and jamming transitions [179] in the perspective of the DPT under cluster merging processes would be interesting. 
Next we discuss the DPT occurring in inter-dependent networks. In this DPT, the order parameter is taken as the fraction of nodes in the mutually connected largest component, which is not the same as the standard ones. The model associated with this DPT was introduced to study cascading-failure dynamics in the inter-dependent systems. Even though the original model was understood analytically, a simpler analytic solution was derived from the viewpoint of epidemic spreading [158]. In this perspective, the DPT can be understood as the emergence of a mutually connected giant cluster in cluster merging processes. Thus, it would be interesting to compare the mechanism of such a DPT with that of the EP model. This investigation would provide a clue to enhance the robustness of inter-dependent networks.

Percolation transitions have served as a platform for understanding phase transitions in non-equilibrium systems. Likewise, the theoretical framework developed to understand the origin of the DPTs occurring in the models i) and ii) is anticipated to serve as a basis for further researches on drastic phase transitions in non-equilibrium complex systems.

\section{Retention capacity and watersheds of landscapes}

Percolation has also been applied to study the properties of real and artificial landscapes. A landscape is typically represented as a digital elevation map (DEM), which consists of a two-dimensional array of regular cells (sites) to which average heights can be associated. By mapping the DEM to a ranked surface, it is possible to identify the sequence of flooded sites when water is dripped over the landscape, filling it from the valleys to the mountains, and letting the water flow out through the open boundaries [92]. In this spirit, one can ask what the maximum volume of water is that can be retained by the landscape. This problem can indeed be mapped to standard percolation [180,181]. For example, consider the simplest case of a two-level random landscape of $L \times L$ sites, with a fraction $p$ sites of unity level and $1-p$ of level zero. The water retention of such a landscape is $R_{2}^{(L)}=L^{2}\left(p-P_{\infty}\right)$, where $P_{\infty}(p)$ is the fraction of sites belonging to the percolation cluster [180]. Since this cluster touches the borders, the water that falls on its sites flows out of the landscape. In the general case of a landscape with equal number of $n$ levels, it was argued [180] that its retention $R_{n}^{(L)}$ can be expressed as,

$$
R_{n}^{(L)}=\sum_{i=1}^{n-1} R_{2}^{(L)}(i / n) .
$$

This expression was also shown to hold for correlated landscapes [182]. Some exact expressions have been found for the retention capacity of finite (and very small) lattice sizes $[180,182]$ but, a general analytic expression is still missing.

In hydrology it is also important to identify the watershed lines dividing the landscape into different drainage basins. When the landscape is flooded from the valleys lakes are formed. As the level of water rises, those lakes start to merge and form even larger lakes. If one merges the lakes under the constraint that no lake percolates, i.e., no lake connects two predefined opposite boundaries of the landscape, one ends up with only two lakes separated by the main watershed line [183]. For random uncorrelated landscapes this line is a fractal of dimension $1.2168 \pm 0.0005$ [184] and its statistical properties are consistent with $\mathrm{SLE}_{\kappa}$, with $\kappa=1.734 \pm 0.005$ [185]. For correlated landscapes, the fractal dimension of the watershed line decreases with the Hurst exponent $[184,186]$. The determination of the watershed line can be mapped to a percolation problem where sites are sequentially occupied (according to their rank 
in the ranked surface) under the constraint that percolation is suppressed [92,187]. Such description reveals a tricritical point at a critical fraction of occupied (flooded) sites and unveils how several seemingly unrelated physical models (e.g., optimal path, optimal path cracks, and polymers in strongly disordered media) fall into the same universality class [92]. The generalization of ranked percolation in three dimensions provides the framework to determine the effective shares when different companies or nations extract either oil, gas, or water, from the same porous formation [188].

\section{Directed percolation}

Let us add finally two comments on models related to directed percolation.

One deals with the (in-)famous "pair contact process with diffusion" (PCPD) in one dimension of space. For a long time this was believed to have continuously varying critical exponents, and yet two recent simulations, Ref. [189] and Ref. [190], still come to opposite conclusions. While the former claim that it is in the directed percolation universality class, the second rules this out.

The second deals with the "parity conserving branching-annihilating random walk" (pcBARW) [191], which mainly differs from directed percolation and the contact process by preserving the "parity" $P(t)=(-1)^{N(t)}$, where $N(t)$ is the number of particles at time $t$. When starting with two initial particles (i.e. in the "even" sector), simulations (the most recent and careful ones being in [192]) suggest that the exponent $\eta$ defined by $N(t) \sim t^{\eta}$ is exactly equal to zero. This cries out for a theoretical explanation, but so far none seems in sight.

\section{Final remarks}

Percolation is a vast subject with more than 80 thousand papers published over the last 60 years according to the ISI Web of Knowledge, and about one paper posted per day on the arXiv related to percolation. Thus, instead of a comprehensive review (which would be an epic journey and impossible in the space available), we decided to rather give a flavor of this fascinating and active field and offer a glimpse at the extensive list of still open questions, following our own interests. We foresee many more years of interesting findings, constantly raising new questions.

We acknowledge financial support from: (NA) the Portuguese Foundation for Science and Technology (FCT), Contracts No. IF/00255/2013 and No. EXCL/FIS-NAN/0083/2012; (BK) the Korean NRF, Grant No. 2010-0015066; and (KJS) the Swiss National Science Foundation, Grant No. P2EZP2-152188. PG thanks Golnoosh Bizhani for interesting discussion and collaboration on cooperative percolation.

\section{References}

1. S.R. Broadbent, J.M. Hammersley, Proc. Cambridge Philos. Soc. 53, 629 (1957)

2. P.J. Flory, J. Am. Chem. Soc. 63, 3083 (1941)

3. P.J. Flory, J. Am. Chem. Soc. 63, 3091 (1941)

4. P.J. Flory, J. Am. Chem. Soc. 63, 3096 (1941)

5. J.W. Essam, Rep. Prog. Phys. 43, 833 (1980)

6. M.E. Fisher, J.W. Essam, J. Math. Phys. 2, 609 (1961)

7. M.E. Fisher, J. Math. Phys. 2, 620 (1961)

8. D. Stauffer, Phys. Rep. 54, 1 (1979) 
9. D. Stauffer, A. Aharony, Introduction to Percolation Theory, 2nd edn. (Taylor and Francis, London, 1994)

10. M. Sahimi, Applications of Percolation Theory (Taylor and Francis, London, 1994)

11. P.W. Kasteleyn, C.M. Fortuin, J. Phys. Soc. Jpn. (Suppl.) 26, 11 (1969)

12. H. Kesten, Commun. Math. Phys. 109, 109 (1987)

13. C. Borgs, J.T. Chayes, H. Kesten, J. Spencer, Commun. Math. Phys. 224, 153 (2001)

14. B. Bollobás, O. Riordan, Percolation (Cambridge University Press, Cambridge, 2006)

15. B. Sapoval, M. Rosso, J.F. Gouyet, J. Phys. Lett. 46, L149 (1985)

16. H. Saleur, B. Duplantier, Phys. Rev. Lett. 58, 2325 (1987)

17. T. Grossman, A. Aharony, J. Phys. A 20, L1193 (1987)

18. S. Smirnov, W. Werner, Math. Res. Lett. 8, 729 (2001)

19. O. Schramm, S. Sheffield, Acta Math. 202, 21 (2009)

20. A. Coniglio, Phys. Rev. Lett. 62, 3054 (1989)

21. J. Cardy, Scaling and Renormalization in Statistical Physics (Cambridge University Press, Cambridge, 1996)

22. M.P.M. den Nijs, J. Phys. A 12, 1857 (1979)

23. B. Nienhuis, E.K. Riedel, M. Schick, J. Phys. A 13, L189 (1980)

24. R.B. Pearson, Phys. Rev. B 22, 2579 (1980)

25. S. Alexander, R. Orbach, J. Physique (Paris) Lett. 43, 625 (1982)

26. P. Grassberger, J. Phys. A 25, 5867 (1992)

27. P. Grassberger, Physica A 262, 251 (1999)

28. H.J. Herrmann, D.C. Hong, H.E. Stanley, J. Phys. A 17, L261 (1984)

29. K.J. Schrenk, N.A.M. Araújo, H.J. Herrmann, Phys. Rev. E 87, 032123 (2013)

30. J. Hoshen, R. Kopelman, Phys. Rev. B 14, 3438 (1976)

31. P.L. Leath, Phys. Rev. B 14, 5046 (1976)

32. M.E.J. Newman, R.M. Ziff, Phys. Rev. Lett. 85, 4104 (2000)

33. H. Kesten, Commun. Math. Phys. 74, 41 (1980)

34. J.C. Wierman, J. Phys. A 17, 1525 (1984)

35. G.R. Grimmett, Percolation, Vol. 321 of Grundlehren der Mathematischen Wissenschaften (Springer, 1999)

36. B. Bollobás, O. Riordan, in An Irregular Mind, Vol. 21 of Bolyai Society Mathematical Studies (Springer, Berlin, 2010)

37. J.L. Cardy, J. Phys. A 25, L201 (1992)

38. R.P. Langlands, C. Pichet, Ph. Pouliot, Y. Saint-Aubin, J. Stat. Phys. 67, 553 (1992)

39. H.T. Pinson, J. Stat. Phys. 75, 1167 (1994)

40. G.M.T. Watts, J. Phys. A 29, L363 (1996)

41. J.J.H. Simmons, P. Kleban, R.M. Ziff, J. Phys. A 40, F771 (2007)

42. O. Schramm, Israel J. Math. 118, 221 (2000)

43. O. Schramm, Electron. Commun. Prob. 6, 115 (2001)

44. S. Smirnov, C. R. Acad. Sci. Paris I 333, 239 (2001)

45. S. Smirnov, Towards conformal invariance of $2 D$ lattice models, in Proceedings of the International Congress of Mathematicians, Madrid, Spain, 2006, edited by M. SanzSolé, J. Soria, J.L. Varona, J. Verdera (European Mathematical Society, Zürich, 2006), p. 1421

46. P. Erdős, A. Rényi, Publ. Math. Inst. Hung. Acad. Sci. 5, 17 (1960)

47. C. Moore, M.E.J. Newman, Phys. Rev. E 62, 7059 (2000)

48. D. Achlioptas, R.M. D'Souza, J. Spencer, Science 323, 1453 (2009)

49. M.F. Sykes, J.W. Essam, Phys. Rev. Lett. 10, 3 (1963)

50. R.M. Ziff, C.R. Scullard, J. Phys. A 39, 15083 (2006)

51. G.R. Grimmett, I. Manolescu, Prob. Thoery Relat. Fields (2013), publ. online

52. R.M. Ziff, C.R. Scullard, J.C. Wierman, M.R.A. Sedlock, J. Phys. A 45, 494005 (2012)

53. F.Y. Wu, J. Phys. C 12, L645 (1979)

54. J.L. Jacobsen, C.R. Scullard, J. Phys. A 46, 075001 (2013)

55. J.L. Jacobsen, J. Phys. A 47, 135001 (2014)

56. S. Miyazima, Bull. Inst. Sci. Technol. Res. 17, 119 (2005) 
57. P. Grassberger, J. Phys. A 32, 6233 (1999)

58. R.M. Ziff, J. Phys. A 32, L457 (1999)

59. R.M. Ziff [arXiv: 1103.3243$]$

60. A. Aharony, D. Stauffer, J. Phys. A 30, L301 (1997)

61. D.R. de Souza, T. Tomé, R.M. Ziff, J. Stat. Mech., P03006 (2011)

62. V. Privman, P.C. Hohenberg, A. Aharony, in Phase Transitions and Critical Phenomena, edited by C. Domb, J.L. Lebowitz, Vol. 14 (Academic, NY, 1991)

63. J.T. Chayes, L. Chayes, G.R. Grimmett, H. Kesten, R.H. Schonmann, Ann. Probab. 17, 1277 (1989)

64. K.A. Seaton, J. Phys. A 34, L759 (2001)

65. G. Delfino, J.L. Cardy, Nucl. Phys. B 519, 551 (1998)

66. J. Cardy, R.M. Ziff, J. Stat. Phys. 110, 1 (2003)

67. R.M. Ziff, C.D. Lorenz, P. Kleban, Physica A 266, 17 (1999)

68. H.N.V. Temperley, E.H. Lieb, Proc. R. Soc. Lond. A 322, 251 (1971)

69. R.M. Ziff, S.R. Finch, V.S. Adamchik, Phys. Rev. Lett. 79, 3447 (1997)

70. R.J. Baxter, H.N.V. Temperley, S.E. Ashley, Proc. R. Soc. Lond. A 358, 535 (1978)

71. M.B. Isichenko, Rev. Mod. Phys. 64, 961 (1992)

72. M. Sahimi, Rev. Mod. Phys. 65, 1393 (1993)

73. A. Coniglio, W. Klein, J. Phys. A 13, 2775 (1980)

74. A. Weinrib, Phys. Rev. B 29, 387 (1984)

75. S. Prakash, S. Havlin, M. Schwartz, H.E. Stanley, Phys. Rev. A 46, R1724 (1992)

76. J. Schmittbuhl, J.P. Vilotte, S. Roux, J. Phys. A 26, 6115 (1993)

77. M. Sahimi, J. Phys. I (France) 4, 1263 (1994)

78. C. Du, C. Satik, Y.C. Yortsos, AIChE J. 42, 2392 (1996)

79. H.A. Makse, S. Havlin, H.E. Stanley, Nature 377, 608 (1995)

80. H.A. Makse, J.S. Andrade Jr., M. Batty, S. Havlin, H.E. Stanley, Phys. Rev. E 58, 7054 (1998)

81. M. Sahimi, Phys. Rep. 306, 213 (1998)

82. H.A. Makse, J.S. Andrade Jr., H.E. Stanley, Phys. Rev. E 61, 583 (2000)

83. A.D. Araújo, A.A. Moreira, H.A. Makse, H.E. Stanley, J.S. Andrade Jr., Phys. Rev. E 66, $046304(2002)$

84. A.D. Araújo, A.A. Moreira, R.N. Costa Filho, J.S. Andrade Jr., Phys. Rev. E 67, $027102(2003)$

85. N. Sandler, H.R. Maei, J. Kondev, Phys. Rev. B 70, 045309 (2004)

86. K.J. Schrenk, N. Posé, J.J. Kranz, L.V.M. van Kessenich, N.A.M. Araújo, H.J. Herrmann, Phys. Rev. E 88, 052102 (2013)

87. A.A. Saberi, Phys. Rev. Lett. 110, 178501 (2013)

88. H. Auradou, K.J. Måløy, J. Schmittbuhl, A. Hansen, D. Bideau, Phys. Rev. E 60, 7224 (1999)

89. J. Schmittbuhl, A. Hansen, H. Auradou, K.J. Måløy, Phys. Rev. E 61, 3985 (2000)

90. J. Kalda, EPL 84, 46003 (2008)

91. J. Kondev, C.L. Henley, D.G. Salinas, Phys. Rev. E 61, 104 (2000)

92. K.J. Schrenk, N.A.M. Araújo, J.S. Andrade Jr., H.J. Herrmann, Sci. Rep. 2, 348 (2012)

93. M.E.J. Newman, R.M. Ziff, Phys. Rev. E 64, 016706 (2001)

94. H. Hu, H.W.J. Blöte, Y. Deng, J. Phys. A 45, 494006 (2012)

95. H.O. Peitgen, D. Saupe (eds.), The Science of Fractal Images (Springer, New York, 1988)

96. K.B. Lauritsen, M. Sahimi, H.J. Herrmann, Phys. Rev. E 48, 1272 (1993)

97. H.A. Makse, S. Havlin, M. Schwartz, H.E. Stanley, Phys. Rev. E 53, 5445 (1996)

98. H.G. Ballesteros, G. Parisi, Phys. Rev. B 60, 12912 (1999)

99. B.D. Malamud, D.L. Turcotte, J. Stat. Plan. Infer. 80, 173 (1999)

100. E.A. Oliveira, K.J. Schrenk, N.A.M. Araújo, H.J. Herrmann, J.S. Andrade Jr., Phys. Rev. E 83, 046113 (2011)

101. B. Ahrens, A.K. Hartmann, Phys. Rev. B 84, 144202 (2011) 
102. P.A. Morais, E.A. Oliveira, N.A.M. Araújo, H.J. Herrmann, J.S. Andrade Jr., Phys. Rev. E 84, 016102 (2011)

103. A. Weinrib, B.I. Halperin, Phys. Rev. B 27, 413 (1983)

104. W. Janke, M. Weigel, Phys. Rev. B 69, 144208 (2004)

105. I. Mandre, J. Kalda, Eur. Phys. J. B 83, 107 (2011)

106. B. Duplantier, Phys. Rev. Lett. 84, 1363 (2000)

107. P. Grassberger, Math. Boisci. 63, 157 (1983)

108. D. Mollison, J. R. Statist. Soc. 39, 283 (1977)

109. J.D. Murray, Mathematical Biology, 3rd edn. (Springer, Berlin, 2005)

110. F.J. Dyson, Commun. Math. Phys. 91, 212 (1969)

111. P.W. Anderson, G. Yuval, D.R. Hamann, Phys. Rev. B 1, 4464 (1970)

112. D.J. Thouless, Phys. Rev. 187, 732 (1969)

113. J.M. Kosterlitz, D.J. Thouless, J. Phys. C 6, 1181 (1973)

114. M. Aizenman, C.M. Newman, Commun. Math. Phys. 107, 611 (1986)

115. P. Grassberger, J. Stat. Mech., P04004 (2013)

116. S. Boettcher, V. Singh, R.M. Ziff, Nat. Commun. 3, 787 (2012)

117. F. Linder, J. Tran-Gia, S.R. Dahmen, H. Hinrichsen, J. Phys. A 41, 185005 (2008)

118. E. Luijten, H.W.J. Blöte, Phys. Rev. Lett. 89, 025703 (2002)

119. P. Grassberger, J. Stat. Phys. 153, 289 (2013)

120. M. Picco [arXiv:1207.1018]

121. T. Blanchard, M. Picco, M.A. Rajabpour, EPL 101, 56003 (2013)

122. N.A.M. Araújo, H.J. Herrmann, Phys. Rev. Lett. 105, 035701 (2010)

123. Y.S. Cho, S. Hwang, H.J. Herrmann, B. Kahng, Science 339, 1185 (2013)

124. R.A. da Costa, S.N. Dorogovtsev, A.V. Goltsev, J.F.F. Mendes, Phys. Rev. Lett. 105, $255701(2010)$

125. O. Riordan, L. Warnke, Science 333, 322 (2011)

126. P. Grassberger, C. Christensen, G. Bizhani, S.W. Son, M. Paczuski, Phys. Rev. Lett. 106, $225701(2011)$

127. G. Bizhani, V. Sood, M. Paczuski, P. Grassberger, Phys. Rev. E 83, 036110 (2011)

128. S.W. Son, G. Bizhani, C. Christensen, P. Grassberger, M. Paczuski, Europhys. Lett. 95, 58007 (2011)

129. C. Christensen, G. Bizhani, S.W. Son, M. Paczuski, P. Grassberger, EPL 97, 16004 (2012)

130. G. Bizhani, P. Grassberger, M. Paczuski, Phys. Rev. E 84, 066111 (2011)

131. H.W. Lau, M. Paczuski, P. Grassberger, Phys. Rev. E 86, 011118 (2012)

132. D.S. Callaway, J.E. Hopcroft, J.M. Kleinberg, M.E.J. Newman, S.H. Strogatz, Phys. Rev. E 64, 041902 (2001)

133. A.L. Barabási, R. Albert, Science 286, 509 (1999)

134. J. Chalupa, P.L. Leath, G.R. Reich, J. Phys. C 12, L31 (1979)

135. J. Adler, Physica A 171, 453 (1991)

136. P.S. Dodds, D.J. Watts, Phys. Rev. Lett. 92, 218701 (2004)

137. P.S. Dodds, D.J. Watts, J. Theor. Biology 42, 232 (2005)

138. H.K. Janssen, M. Müller, O. Stenull, Phys. Rev. E 70, 026114 (2004)

139. G. Bizhani, M. Paczuski, P. Grassberger, Phys. Rev. E 86, 011128 (2012)

140. A.L. Barabási, H.E. Stanley, Fractal Concepts in Surface Growth (Cambridge University Press, New York, 1995)

141. A.V. Goltsev, S.N. Dorogovtsev, J.F.F. Mendes, Phys. Rev. E 73, 056101 (2006)

142. P. Le Doussal, K.J. Wiese, P. Chauve, Phys. Rev. B 66, 174201 (2002)

143. B. Drossel, K. Dahmen, Eur. Phys. J. B 3, 485 (1998)

144. N.J. Zhou, B. Zheng, Phys. Rev. E 82, 031139 (2010)

145. X.P. Qin, B. Zheng, N.J. Zhou, J. Phys. A 45, 345005 (2012)

146. M. Aizenman, J. Wehr, Phys. Rev. Lett. 62, 2503 (1989)

147. J.P. Sethna, K.A. Dahmen, O. Perković [arXiv: cond-mat/0406320]

148. M. Singer, Introduction to Syndemics: A Critical Systems Approach to Public and Community Health (John Wiley \& Sons, Hoboken, 2009)

149. J.F. Brundage, J.D. Shanks, Emerg. Infect. Dis. 14, 1193 (2008) 
150. W. Oei, H. Nishiura, Comput. Math. Meth. Med. 2012, 124861 (2012)

151. M.S. Sulkowski, J. Hepatology 48, 353 (2008)

152. S.K. Sharma, A. Mohan, S. Kadhivaran, Indian J. Med. Res. 121, 550 (2005)

153. L. Chen, W. Cai, F. Ghanbarnejad, P. Grassberger, Europhys. Lett. 104, 50001 (2013)

154. W. Cai, L. Chen, F. Ghanbarnejad, P. Grassberger (2014) (published)

155. S.V. Buldyrev, R. Parshani, G. Paul, H.E. Stanley, S. Havlin, Nature 464, 1025 (2010)

156. R. Parshani, S.V. Buldyrev, S. Havlin, Phys. Rev. Lett. 105, 048701 (2010)

157. J. Gao, S.V. Buldyrev, H.E. Stanley, S. Havlin, Nat. Phys. 8, 40 (2011)

158. S.W. Son, G. Bizhani, C. Christensen, P. Grassberger, M. Paczuski, Europhys. Lett. 97, 16006 (2012)

159. B. Bollobás, Graph Theory and Combinatorics: Proc. Cambridge Combinatorial Conference in Honour of Paul Erdős (Academic Press, New York, 1984)

160. S.N. Dorogovtsev, A.V. Goltsev, J.F.F. Mendes, Phys. Rev. Lett. 96, 040601 (2006)

161. R.M. Ziff, Phys. Rev. Lett. 103, 045701 (2009)

162. Y.S. Cho, J.S. Kim, J. Park, B. Kahng, D. Kim, Phys. Rev. Lett. 103, 135702 (2009)

163. E.J. Friedman, A.S. Landsberg, Phys. Rev. Lett. 103, 255701 (2009)

164. R.M. D'Souza, M. Mitzenmacher, Phys. Rev. Lett. 104, 195702 (2010)

165. J. Nagler, A. Levina, M. Timme, Nat. Phys. 7, 265 (2011)

166. R.M. Ziff, Science 339, 1159 (2013)

167. R.F.S. Andrade, H.J. Herrmann, Phys. Rev. E 88, 042122 (2013)

168. Y.S. Cho, B. Kahng [arXiv: 1404.4470]

169. R.M. Ziff, Phys. Rev. E 82, 051105 (2010)

170. Y.S. Cho, B. Kahng, D. Kim, Phys. Rev. E 81, 030103(R) (2010)

171. T. Bohman, A. Frieze, N.C. Wormald, Random Struct. Algorithms 25, 432 (2004)

172. W. Chen, R.M. D'Souza, Phys. Rev. Lett. 106, 115701 (2011)

173. W. Chen, Z. Zheng, R.M. D'Souza, EPL 100, 66006 (2012)

174. K.J. Schrenk, A. Felder, S. Deflorin, N.A.M. Araújo, R.M. D'Souza, H.J. Herrmann, Phys. Rev. E 85, 031103 (2012)

175. K. Panagiotou, R. Spöhel, A. Steger, H. Thomas, Electron. Notes Discr. Math. 38, 699 (2011)

176. R. Zhang, W. Wei, B. Guo, Y. Zhang, Z. Zheng, Physica A 392, 1232 (2013)

177. W. Chen, J. Nagler, X. Cheng, X. Jin, H. Shen, Z. Zheng, R.M. D'Souza, Phys. Rev. E 87, 052130 (2013)

178. J. Gómez-Gardeñes, S. Gómez, A. Arenas, Y. Moreno, Phys. Rev. Lett. 106, 128701 (2011)

179. P. Echenique, J. Gómez-Gardeñes, Y. Moreno, Europhys. Lett. 71, 325 (2005)

180. C.L. Knecht, W. Trump, D. ben-Avraham, R.M. Ziff, Phys. Rev. Lett. 108, 045703 (2012)

181. S.K. Baek, B.J. Kim, Phys. Rev. E 85, 032103 (2012)

182. K.J. Schrenk, N.A.M. Araújo, R.M. Ziff, H.J. Herrmann [arXiv: 1403.2082v1]

183. E. Fehr, J.S. Andrade Jr., S.D. da Cunha, L.R. da Silva, H.J. Herrmann, D. Kadau, C.F. Moukarzel, E.A. Oliveira, J. Stat. Mech., P09007 (2009)

184. E. Fehr, K.J. Schrenk, N.A.M. Araújo, D. Kadau, P. Grassberger, J.S. Andrade Jr., H.J. Herrmann, Phys. Rev. E 86, 011117 (2012)

185. E. Daryaei, N.A.M. Araújo, K.J. Schrenk, S. Rouhani, H.J. Herrmann, Phys. Rev. Lett. 109, $218701(2012)$

186. E. Fehr, D. Kadau, J.S. Andrade Jr., H.J. Herrmann, Phys. Rev. Lett. 106, 048501 (2011)

187. R.F.S. Andrade, H.J. Herrmann, Phys. Rev. E 87, 042113 (2013)

188. K.J. Schrenk, N.A.M. Araújo, H.J. Herrmann, Sci. Rep. 2, 751 (2012)

189. R.D. Schram, G.T. Barkema, J. Stat. Mech., P03009 (2012)

190. S.C. Park [arXiv:1202.6024v2]

191. J. Marro, R. Dickman, Nonequilibrium Phase Transitions in Lattice Models (Cambridge University Press, Cambridge, 1998)

192. S.C. Park, J. Korean Phys. Soc. 62, 469 (2013) 